\title{
Desenvolvimento de um sistema automatizado para medição de impedância elétrica
}

\author{
Development of an automated system for measuring electrical impedance \\ Joseanne Cristina Viana, Francisco Arnold* \\ Faculdade de Tecnologia, Universidade Estadual de Campinas, Limeira, SP, Brasil
}

Recebido em 18 de Agosto, 2016. Revisado em 19 de Setembro, 2016. Aceito em 29 de Outubro, 2016.

\begin{abstract}
A medição de impedâncias elétricas é um procedimento bastante comum em laboratórios de Física. Convencionalmente, por meio de medições de tensão elétrica um circuito divisor de tensão se pode determinar a impedância elétrica de um dispositivo qualquer. Todavia, este procedimento permite que as impedâncias só possam ser medidas em faixas de valores restritas. Em componentes, como alguns transdutores piezelétricos, que apresentam larga faixa de magnitude de impedância, os resultados obtidos com este procedimento não são satisfatórios. Neste trabalho é apresentado um circuito eletrônico simples, de baixo custo, com fácil compreensão de operação por estudantes com pouco conhecimento sobre eletrônica e que amplia a faixa de determinação das impedâncias. O sistema foi testado com transdutores piezelétricos e os resultados obtidos comparados com o de um analisador de impedâncias comercial. Os resultados mostraram que é possível medir impedâncias entre dezenas de $\Omega$ e dezenas de $\mathrm{k} \Omega$ satisfatoriamente.
\end{abstract}

Palavras-chave: LabView, Piezelétrico, Circuito Eletrônico

\begin{abstract}
The measurement of electrical impedance is a common procedure in physics laboratories. Conventionally, by means of voltage measurements a voltage divider circuit can determine the electrical impedance of any device. However, this procedure allows the impedances can be measured only in limited ranges of values. In components as some piezoelectric transducers, which have large impedance magnitude band, the results obtained with this procedure are not satisfactory. This paper presents a simple electronic circuit, low cost, easy to understand operation by students with low knowledgement in electronics and broadens the range of determination of impedances. The system was tested with piezoelectric transducers and the results compared with a commercial impedance analyzer. The results have shown that it is possible to measure impedances between tens of $\Omega$ and tens of $\mathrm{k} \Omega$ satisfactorily.
\end{abstract}

Keywords: LabView, Piezoelectric, Electronic Circuit

\section{Introdução}

O emprego de recursos tecnológicos visando criar maior dinamismo e exatidão em experimentos de física tem atraído especial atenção nos últimos anos nas publicações voltadas ao ensino. A massificação da utilização de recursos eletrônicos [1,2], sensores [3], vídeos [4] e produtos ligados a informática [5-7] no ensino é uma decorrência, entre outros motivos, da maior acessibilidade a dispositivos e softwares para automatização dos experimentos. Os equipamentos eletrônicos inseridos nas práticas tradicio-

*Endereço de correspondência: arnold@ft.unicamp.br nais atrai a atenção dos alunos pelo fascínio que desperta em relação à praticidade e à velocidade na obtenção e ao processamento de resultados, além de estimular o espírito inventivo na concepção de outros experimentos.

Medir impedâncias elétricas é uma prática bastante comum em laboratórios de física de ensino e de pesquisa. Nas práticas de ensino, medir impedâncias elétricas é conteúdo obrigatório na exploração de propriedades de circuitos elétricos em correntes alternadas, usualmente ensinada na disciplina Física IV [8-10]. Em pesquisa, a medição de impedâncias elétricas tem maior abrangência pois, em grande 
parte dos casos, consiste na caracterização de dispositivos de diferentes naturezas, como componentes elétricos e eletrônicos e sensores. De um modo geral, os procedimentos dos experimentos abarcam a determinação da magnitude e da fase (ou das componentes real e imaginária) da impedância elétrica de um dispositivo qualquer numa faixa de frequências.

Há, basicamente, dois caminhos para se medir as impedâncias elétricas. Pode-se empregar um analisador de impedâncias comercial para obtenção dos espectros de frequência e fase. Os analisadores de impedância comerciais são equipamentos de larga complexidade tecnológica, com uma gama de aplicações muito ampla, grandes dimensões físicas e de custo muito elevado. Portanto podem não ser viáveis para um laboratório de ensino ou mesmo para serem empregado em finalidades específicas ou atividades de campo.

Outra forma de medir impedâncias elétricas é por meio do circuito esquematizado na Figura 1. Esta metodologia convencional [11] consiste em usar um divisor de tensão elétrica em que o elemento que se deseja conhecer a impedância $\left(Z_{t}\right)$ está em série com um resistor shunt $(R)$. O divisor é alimentado com sinais elétricos senoidais $(v)$ de um gerador de funções, de modo que seja possível ajustar a frequência na faixa de valores desejada e medir, usando-se um osciloscópio, as tensões de entrada $\left(v_{1}\right)$ e nos terminais do shunt $\left(v_{2}\right)$. A partir dessas medições, empregandose análise de circuitos elétricos elementar se pode determinar a magnitude da impedância elétrica do dispositivo. A componente de fase da impedância elétrica também pode ser determinada por meio das medições dos tempos de atraso ou adiantamento de $v_{1}$ em relação a $v_{2}$. Esta prática é bastante comum nos laboratórios de ensino e de pesquisa, devido ao emprego de equipamentos de uso genérico nos laboratórios e de baixo custo.

Todavia, empregar esta metodologia para medições em que o módulo das impedâncias varia largamente, nem sempre é adequado. Por exemplo, quando o valor da resistência shunt for muito pequeno em relação a impedância elétrica do dispositivo a ser caracterizado $\left(R<<\left|Z_{t}\right|\right)$, podem ocorrer dificuldades em medir a baixa amplitude de tensão elétrica entre os terminais do shunt devido às limitações de escala típicas de um osciloscópio de uso geral e o surgimento de ruídos. Numa situação inversa, quando a resistência do shunt é muito maior que a impedância elétrica do dispositivo em teste $\left(R>>\left|Z_{t}\right|\right)$, as va-

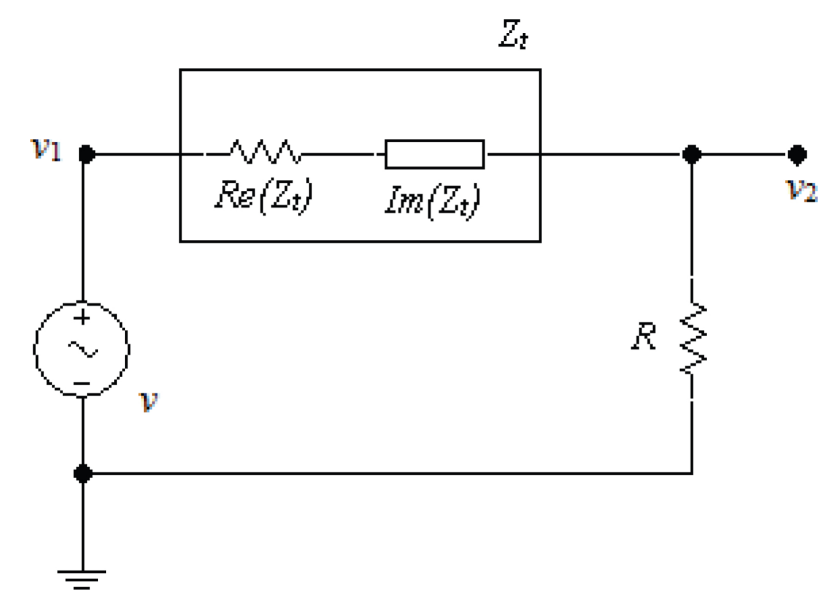

Figura 1: Diagrama esquemático de um divisor de tensão como uma configuração simples para determinação de impedâncias elétricas.

riações de tensão podem não ser corretamente avaliadas devido a baixa resolução dos equipamentos de medida. Além disso, quando a tensão medida no shunt apresenta amplitudes reduzidas, ocorre a inserção de ruídos que deformam o sinal e dificultam a determinação da diferença de fase entre os sinais $v_{1}$ e $v_{2}$.

Em sinais de baixa amplitude a utilização de amplificadores para o sinal de corrente pode ser feita para superar essas dificuldades de medição, porém estes podem ir a saturação quando o sinal se tornar mais elevado. Além disso, o uso de amplificadores de ganho elevado pode contribuir para a redução da banda de frequências a ser utilizada.

Variações do sinal de corrente da ordem de algumas décadas são típicas quando se mede a impedância de transdutores piezelétricos. Os transdutores piezelétricos são apresentados em diferentes modelos que dependem de sua aplicação [12]. Particularmente, transdutores piezelétricos usados em aplicações com potências elevadas (da ordem de dezenas de $\mathrm{W}$ ) apresentam variações da magnitude da impedância da ordem de dezenas de $\mathrm{k} \Omega$. Sendo assim, a metodologia convencional deve ser pouco efetiva para a determinação das impedâncias devido a larga faixa de variação alcançada ao longo das frequências.

Na literatura científica são encontrados alguns trabalhos que tratam do desenvolvimento de sistemas de medição de impedâncias elétricas. Fishman [13] apresentou um sistema eletrônico digital integrado a um computador juntamente com resultados experimentais de magnitude e fase da impedância, 
porém restrito a valores de frequência da ordem de centenas de Hz. Peiars et al. [14] e Batista et al. [15] apresentaram soluções para medir impedâncias em testes de monitoramento da integridade mecânica (Structural Health Monitoring - SHM) de materiais usando-se dispositivos piezelétricos. Eles empregaram a configuração convencional do divisor resistivo com o resistor shunt ligado a um amplificador operacional. Os resultados apresentados nesses artigos se mostraram precisos, porém restritos a variações de centenas de ohms, típicas dos transdutores aplicados em SHM. O ajuste das resistências de uma ponte foi utilizada em [16] por meio de controles em potenciômetros digitais em aplicações de SHM, mas os resultados foram apresentados como impedâncias relativas e com variações de valores muito inferiores às dos piezelétricos para alta potência.

Outra forma de medir correntes para determinar a impedância consiste no uso de sensores magnéticos. A utilização de sensores magnéticos [17] apresenta limitações causadas pela interferência da sua componente de reatância indutiva no circuito sob frequências elevadas e/ou quando o elemento em teste apresentar valores de reatâncias próximos ao assumidos pelo sensor. Outros sistemas para medição mais sofisticados são encontrados em [18,19]. Contudo, estes sistemas se utilizam de técnicas eletrônicas que fogem do escopo deste artigo, qual seja de encontrar uma solução de um problema comum nos laboratórios de física com didática e simplicidade e que possa ser construído com facilidade.

Neste trabalho é proposta uma adaptação a configuração apresentada na Figura 1 com intuito de superar algumas dificuldades de medição quando os valores de $R$ e $Z_{t}$ são díspares. Essa adaptação é baseada no circuito mostrado na Figura 2. Ela consiste em realizar a medição da tensão no componente em teste ao invés do resistor shunt e fazer uma transformação linear da faixa de valores medidos em outra faixa que possa ser adequadamente medida por um osciloscópio ou módulo digital. O resultado dessa transformação irá modificar os valores extremos de tensão acusados anteriormente como limitantes e permitir que a medição seja feita em condições adequadas de operação precisa dos medidores.

O objetivo do trabalho é apresentar o desenvolvimento de um circuito de baixo custo e operação simplificada e de uma metodologia que possa ser utilizada e compreendida por alunos dos semestres inici-

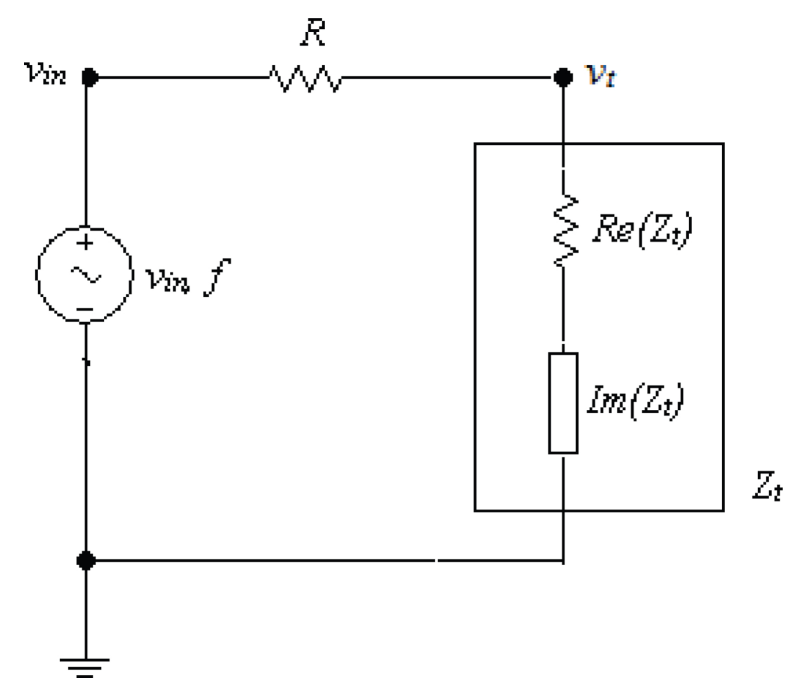

Figura 2: Diagrama esquemático do divisor de tensão utilizado no sistema desenvolvido.

ais de um curso de Física, Engenharia ou afins. Este circuito deve ser útil para que se meçam impedâncias elétricas em uma faixa larga de magnitudes, como as apresentadas por transdutores piezelétricos usados em aplicações de alta potência.

\section{Teoria de circuitos}

Nesta seção é apresentada a teoria de circuitos, baseada em álgebra fasorial [20,21], que explica o procedimento utilizado para a determinação da impedância elétrica $Z_{t}$. Na Figura 2 é apresentado o circuito que será utilizado para a análise. Como pode ser visto nesta figura, a impedância de teste é $Z_{t}$ possui as componentes real e imaginária dadas por $\operatorname{Re}\left(Z_{t}\right)=R_{t}$ e $\operatorname{Im}\left(Z_{t}\right)=X_{t}$, respectivamente.

Com o embasamento fasorial utilizado para a análise de circuitos, definem-se $V_{i n}$ e $V_{t}$ como as amplitudes das tensões $v_{i n}$ e $v_{t}$. A impedância total, $Z$, "vista"pela fonte $v_{i n}$, representada nas notações complexa e fasorial, é dada pela Equação 1.

$$
Z=\left(R+R_{t}\right)+j X_{t}=|Z| \angle \theta
$$

onde $R$ é a resistência do divisor de tensão, $R_{t}$ e $X_{t}$ são as partes real e imaginária da impedância do componente medido, $\theta$ é o ângulo de fase da impedância e $j$ é a unidade complexa.

A impedância a ser determinada é $Z_{t}$, é descrita pela Equação (2), tanto na notação complexa como fasorial.

$$
Z_{t}=R_{t}+j X_{t}=\left|Z_{t}\right| \angle \theta_{t}
$$


A amplitude da tensão elétrica medida no resistor $\mathrm{R}$ é calculada usando-se a teoria de circuitos elétricos pela Equação (3)

$$
V_{R}=V_{i n} \frac{R}{\sqrt{\left(R+R_{t}\right)^{2}+X_{t}^{2}}}
$$

A diferença de fase entre $v_{i n}$ e a corrente do circuito é a mesma diferença entre a $v_{i n}$ e $v_{R}$ e pode ser calculada com a Equação (4)

$$
\tan \theta=\frac{X_{t}}{R+R_{t}}
$$

O ângulo de fase $\theta$ depende da natureza do efeito reativo de $Z_{t}$, que pode ser capacitivo $\left(X_{t}<0\right)$ ou indutivo $\left(X_{t}>0\right)$. O diagrama de fasores referentes a impedância do circuito apresenta as duas configurações que são mostradas na Figura 3.

Adotando-se a fase da tensão de entrada $v_{i n}$ como $0, v_{\text {in }}=V_{i} \angle 0^{\circ}$, determina-se o fasor da corrente $I$ pela Equação 5. Na Figura 3 também está inserida a representação fasorial da corrente do circuito.

$$
I=\frac{V_{\text {in }} \angle 0}{Z \angle \theta}=\frac{V_{\text {in }}}{Z} \angle-\theta
$$

Com isso, os fasores das tensões $v_{R}$ e $v_{t}$ são calculados com as Equações 6 e 7 .

$$
\begin{gathered}
v_{R}=I R=\frac{V_{i n}}{Z} \angle-\theta \\
v_{t}=I Z_{t} \angle \theta_{t}=\frac{V_{i n}}{Z} R \angle\left(\theta_{t}-\theta\right)
\end{gathered}
$$

Conforme já conhecido da teoria de circuitos, as fases entre das impedâncias são restritas a ângulos de $90^{\circ}$. Portanto, $-90^{\circ}<\theta_{t}<90^{\circ}$ e $-90^{\circ}<\theta<90^{\circ}$.
A tensão $v_{t}$ possui um ângulo de fase $\theta_{v t}=\theta_{t}-\theta$ em relação a $v_{i n}$. Com isso, os fasores dos diagramas da Figura 3 sendo multiplicados por I são rotacionados e produzem o diagrama fasorial das tensões mostrado na Figura 4

Adotando-se um procedimento experimental em que são medidos $V_{i n}, V_{t}$ e $\theta_{v t}$, determina-se $V_{R}$ usando a lei dos cossenos (Equação 8) nos triângulos formados nos diagramas fasoriais da Figura 4 .

$$
V_{R}^{2}=V_{i n}^{2}+V_{t}^{2}-2 V_{i n} V_{t} \cos \theta_{v t}
$$

Aplicando-se novamente a lei dos cossenos nos fasores da Figura 4, obtem-se o ângulo $\theta$ com a Equação 9

$$
\cos \theta=\frac{V_{t}^{2}-V_{R}^{2}-V_{i n}^{2}}{-2 V_{R} V_{\text {in }}}
$$

Com os resultados obtidos por meio das Equações 8 e 9 pode-se calcular a $Z_{t} \angle \theta_{t}$. A magnitude de $Z_{t}$ é obtida pela igualdade das correntes em $Z_{t}$ e $R$ (Equação 10). A fase é obtida somando-se $\theta_{t}=\theta_{v t}+\theta$

$$
Z_{t}=R \frac{V_{t}}{V_{R}}
$$

\section{Descrição do circuito}

Como é sabido, em um divisor de tensão em que os valores dos componentes possuem resistência (ou impedância) díspares, a voltagem sobre o elemento de menor valor é muito pequena. Medições de tensões na ordem de dezenas de $\mathrm{mV}$, em condições não preparadas especificamente para este fim, podem ser comprometidas pelo surgimento de interferências indesejáveis. Além disso, muitos equipamentos, como

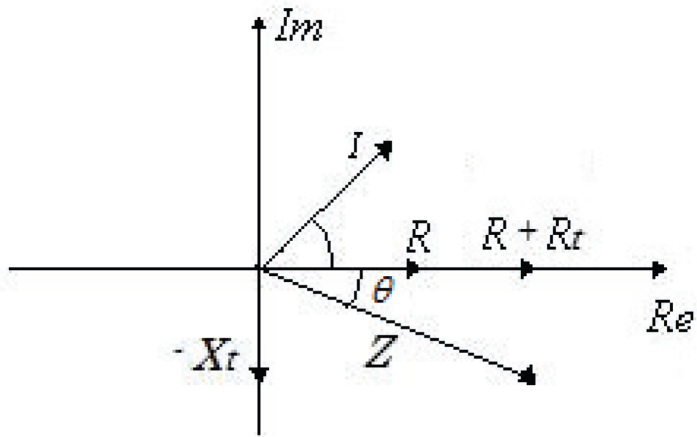

(a)

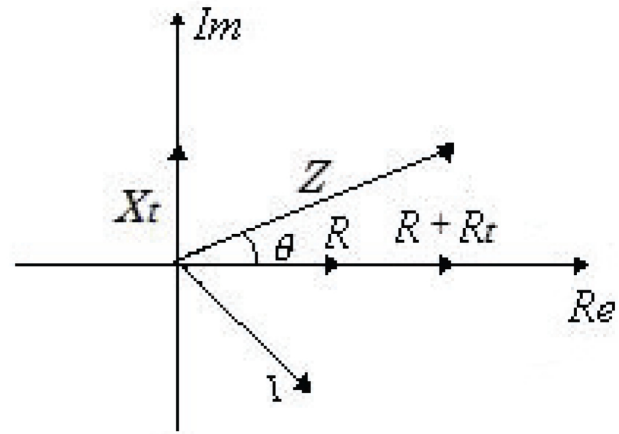

(b)

Figura 3: (a) Representação fasorial entre corrente / e Impedância $Z$ para circuitos capacitivos $\left(X_{t}<0\right)$. (b) Representação fasorial entre corrente $I$ e Impedância $Z$ para circuitos indutivos $\left(X_{t}>0\right)$. 


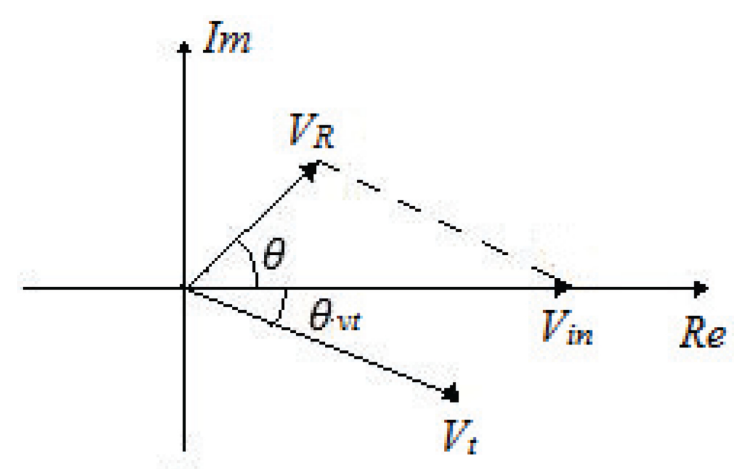

(a)

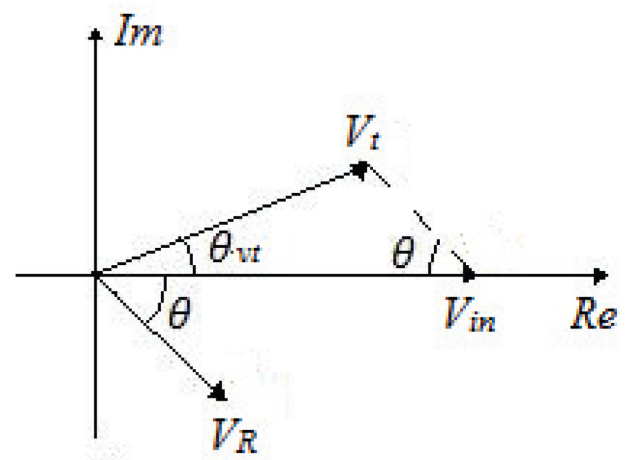

(b)

Figura 4: (a) Representação fasorial das tensões para circuitos capacitivos $\left(X_{t}<0\right)$.(b) Representação fasorial das tensões para circuitos indutivos $\left(X_{t}>0\right)$

osciloscópios e placas digitalizadoras comuns, que medem voltagens não operam satisfatoriamente sob amplitudes dessa ordem. O circuito desenvolvido neste trabalho visa superar as dificuldades causadas por essa situação por meio da amplificação dessas tensões para níveis em que as medições possam ser menos afetadas pelas eventuais interferências e o medidor opere sem restrições em plenitude.

A operação do circuito é baseada numa transformação linear em que a tensão medida no dispositivo em teste é somada a um nível de tensão mínimo e amplificada. O resultado dessa operação deve garantir um valor mínimo de tensão de saída o qual possa ser medido sem maiores dificuldades técnicas.
O diagrama esquemático do circuito proposto no trabalho é mostrado na Figura 5. Na Figura 5 foram omitidos os terminais de alimentação dos amplificadores operacionais e os capacitores de bypass normalmente conectados entre os terminais $+\mathrm{V}$ e -V e o terra da fonte de alimentação.

O divisor de tensão de entrada é composto por $R 1$ e $Z_{t} . Z_{t}$ é o elemento a ter sua impedância elétrica determinada. Conforme a frequência do sinal elétrico fornecido pelo gerador de sinais, $v_{i n}$, a queda de tensão elétrica em $Z_{t}$ é modificada. Dessa forma, a variação entre os valores de $Z_{t \min }$ e $Z_{t \text { max }}$, prevista no projeto, deve produzir uma variação na amplitude da tensão na entrada não inversora de AO1 entre $V_{t \_ \text {min }}$ e $V_{t \_m a x}$. O valor de $V_{t \_m i n}$ deve apresentar

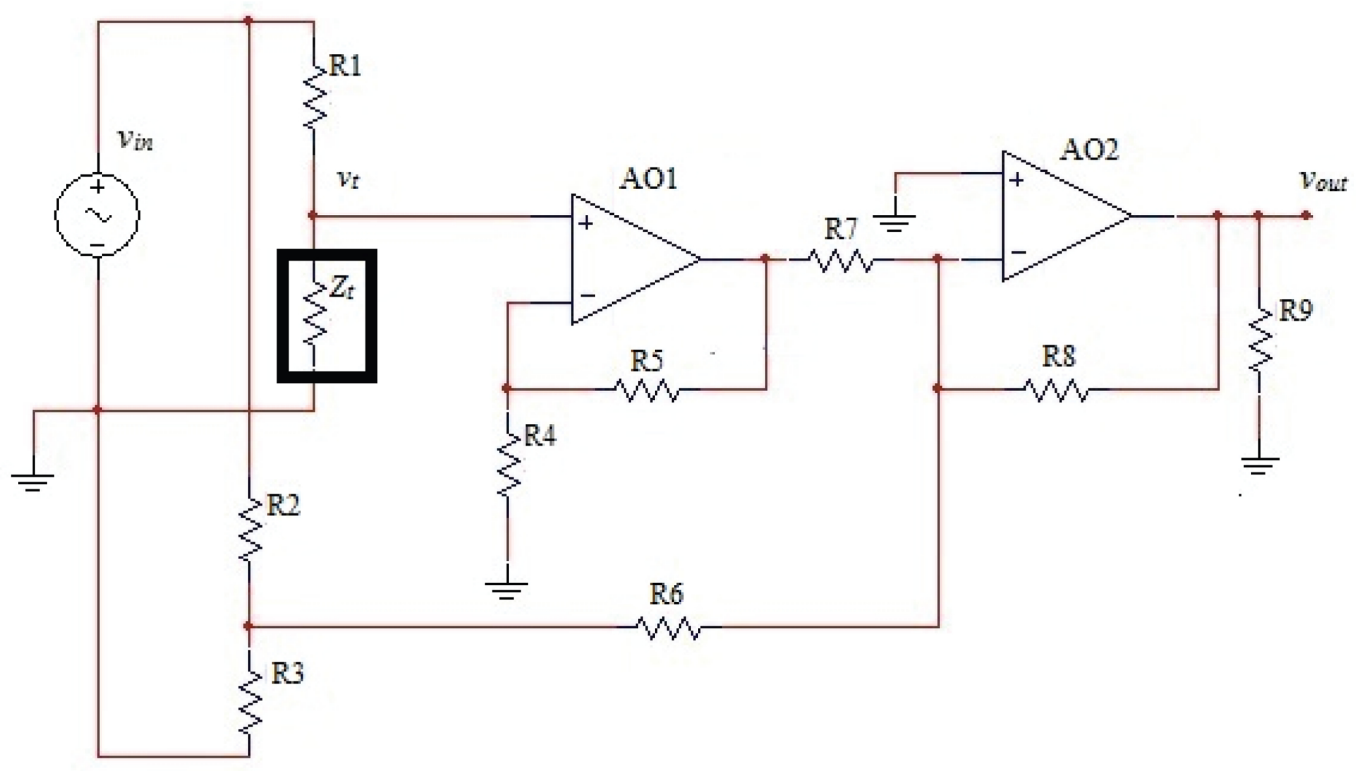

Figura 5: Diagrama esquemático do circuito eletrônico que amplifica os sinais e modifica a sensibilidade da medição da corrente para cálculo da impedância elétrica. 
problemas de medição caso o valor de $Z_{t \text { min }}$ seja muito menor que $R 1$. Nesse caso, é necessário que o valor de $V_{t}$ seja amplificado. Contudo, por outro lado, esta amplificação pode gerar níveis de tensão mais elevados e uma possível saturação do amplificador quando o valor de $Z_{t}$ for mais elevado.

A solução para este problema consiste em aplicar uma transformação linear na tensão de saída do divisor de tensão. Dessa forma, a amplitude da tensão na saída do amplificador operacional AO2 deve ser dada pela Equação (11)

$$
V_{\text {out }}=A V_{t}+B
$$

onde $A$ e $B$ são constantes a serem determinadas;

$V_{t}$ é a amplitude da tensão em $Z_{t}$ que varia entre valores estabelecidos pela relação de $R 1$ e $Z_{t}$;

$V_{\text {out }}$ é a amplitude da tensão de saída resultante de uma amplificação adequada dos valores extremos de $V_{t}$.

A operação definida pela Equação 11 é feita por um circuito composto pelos amplificadores operacionais: AO1 que promove a multiplicação $A v_{t}$ e AO2 que executa a soma $A v_{t}+B$. A teoria sobre amplificadores operacionais e suas configurações típicas são apresentadas em [22].

A determinação de $A$ e $B$ é feita considerando os extremos das faixas de tensão previstas no projeto. Assim se obtém um sistema de equações lineares dado em (12):

$$
\begin{aligned}
V_{\text {out } \min } & =A V_{t \text { min }}+B \\
V_{\text {out } \max } & =A V_{t \text { max }}+B
\end{aligned}
$$

Os resistores $R 2$ e $R 3$ compõem um divisor de tensão que são definidos a partir do valor de $B$. $O$ valor de $B$ é uma constante calculada pela Equação 13.

$$
B=\frac{R 4}{R 3+R 4} V_{i n}
$$

$R 4$ e $R 5$ são os resistores que definem o ganho do amplificador operacional AO1 na configuração não inversora, dado pela Equação 14 e que fornecem a componente $A v_{t}$ para o circuito somador.

$$
A=1+\frac{R 5}{R 4}
$$

\section{Desenvolvimento do projeto e metodologia}

No projeto que foi desenvolvido neste trabalho foram estabelecidos os seguintes parâmetros: $v_{i n}=28 \mathrm{~V}_{\mathrm{pp}}$,
$Z_{t \min }=10 \Omega, Z_{t \max }=100 \mathrm{k} \Omega, v_{\text {out_min }}=0,3 \mathrm{~V}_{\mathrm{pp}}$ e $v_{\text {out_max }}=25,0 \mathrm{~V}_{\mathrm{pp}}$. A alimentação é simétrica de $\pm 18 \mathrm{~V}$ e entre os terminais da fonte e o terra foram usados capacitores de bypass de $100 \mathrm{nF}$.

A obtenção de $v_{\text {in }}$ foi feita a partir de um gerador de funções Tektronix AFG3021C. Na saída deste gerador foi ligado um amplificador operacional na configuração não inversora que forneceu os $28 \mathrm{~V}_{\mathrm{pp}}$

$\mathrm{O}$ resistor $R 1$ é de $15 \mathrm{k} \Omega$ e foi escolhido por se tratar de um valor intermediário da faixa de impedâncias que o elemento a ser testado poderia apresentar. Este critério foi adotado pois pretendese utilizar o medidor para determinar impedâncias de transdutores piezelétricos de potência que apresentam, em geral, variações de magnitude entre dezenas de $\Omega$ e dezenas de $\mathrm{k} \Omega$. Com isso, as tensões $v_{\text {in_min }}$ e $v_{\text {in_max }}$ são de $0,019 \mathrm{~V}_{\mathrm{pp}}$ e $24,3 \mathrm{~V}_{\mathrm{pp}}$, respectivamente. Resolvendo-se o sistema de equações (12) obteve-se $A=1,02$ e $B=0,28 \mathrm{~V}$

A Tabela I apresenta os valores calculados a partir das premissas do projeto. O circuito foi simulado, construído e calibrado antes da realização dos testes.

Os testes foram realizados em transdutores piezelétricos de potência produzidos pela Beijing Chengcheng Weiye Ultrasonic Science and Technology Co. Ltda. Os transdutores possuem frequências de ressonância nos seus modos fundamentais em torno de $27 \mathrm{kHz}, 28 \mathrm{kHz}$ e $38 \mathrm{kHz}$ e serão denominados P1, P2 e P3, respectivamente. As impedâncias desses transdutores foram determinadas na faixa de $10 \mathrm{kHz}$ a $70 \mathrm{kHz}$ usando-se um analisador de impedâncias HP4294A, produzido pela Agilent. O analisador fornece as curvas do módulo e da fase da impedância em função da frequência que servirão de referência para validação do sistema desenvolvido.

As medições da tensão de entrada e saída foram feitas por um módulo digital NI USB51-32 da National Instruments que realiza funções de medição de tensão similares a de um osciloscópio. As configurações do módulo digitalizador e do gerador de funções, assim como o controle da varredura dos processos, foram feitas por um programa desenvolvido na plataforma LabView (National Instruments). Este mesmo programa possui rotina para determinar magnitude e fase da impedância. A Figura 6 mostra um diagrama de blocos do sistema completo.

A interface com o usuário desenvolvida no LabView é mostrada na Figura 7.

Os resultados são apresentados em gráficos comparativos contendo as curvas de magnitude e fase da 
Tabela 1: Valores das resistências dos resistores calculados (valores comerciais aproximados). Todos os resistores possuem potencias nominais de $1 / 4 \mathrm{~W}$ e tolerância de $\pm 5 \%$.

\begin{tabular}{lllllllll}
\hline $\mathrm{R} 1$ & $\mathrm{R} 2$ & $\mathrm{R} 3$ & $\mathrm{R} 4$ & $\mathrm{R} 5$ & $\mathrm{R} 6$ & $\mathrm{R} 7$ & $\mathrm{R} 8$ & $\mathrm{R} 9$ \\
\hline $15 \mathrm{k} \Omega$ & $10 \mathrm{k} \Omega$ & $100 \Omega$ & $10 \mathrm{k} \Omega$ & $150 \Omega$ & $1 \mathrm{k} \Omega$ & $1 \mathrm{k} \Omega$ & $1 \mathrm{k} \Omega$ & $10 \mathrm{k} \Omega$ \\
\hline
\end{tabular}

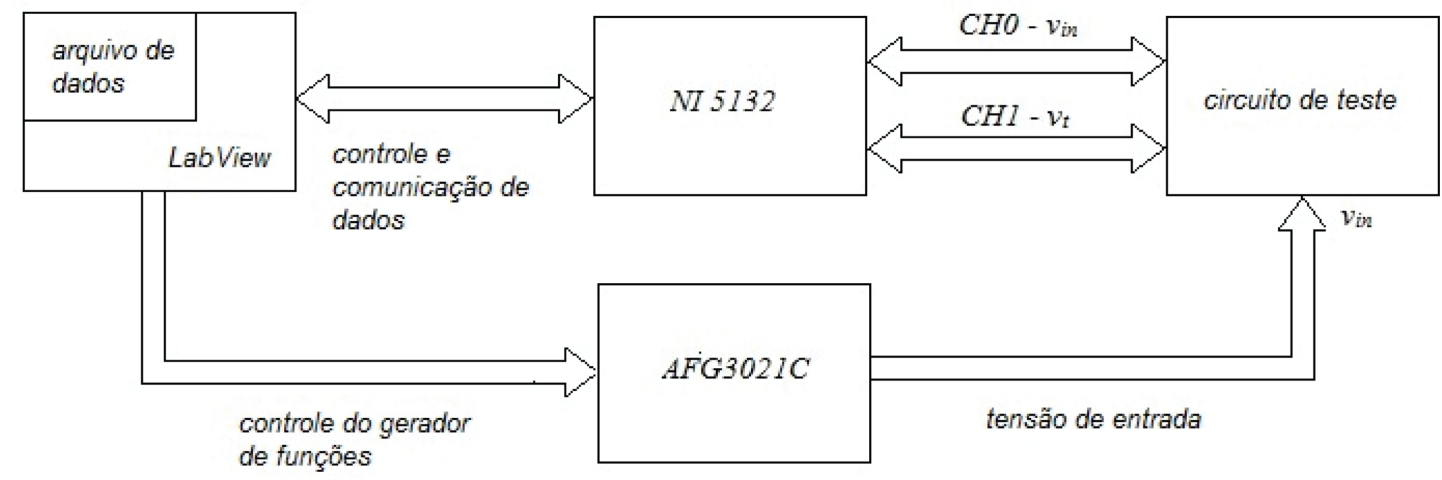

Figura 6: Diagrama de blocos do sistema completo.

impedância do sistema desenvolvido e do HP4294A. Cada curva contem 1000 pontos.

\section{Resultados e discussões}

Os resultados obtidos com os transdutores P1, P2 e P3 são mostrados nas Figuras 8, 9 e 10, respectivamente.

De acordo com o esperado, a tensão no elemento $Z_{t}$ pode ser medida numa faixa entre 0,019 e 24,3 Vpp e, com isso, determinar as impedâncias numa faixa larga de valores. Este ponto representa uma vantagem em relação ao circuito da Figura 1.Uma tensão de $28 \mathrm{Vpp}$ aplicada ao circuito da Figura 1 , considerando um resistor shunt com valores em torno de uma centena de $\Omega$, geraria elevadas demandas de potência.

A medição direta no divisor de tensão com o módulo digital NI USB51-32 não é viável quando as tensões medidas são inferiores a $150 \mathrm{mVpp}$, aproximadamente. Dessa forma, é necessário que seja realizada uma elevação da tensão a ser medida. Essa

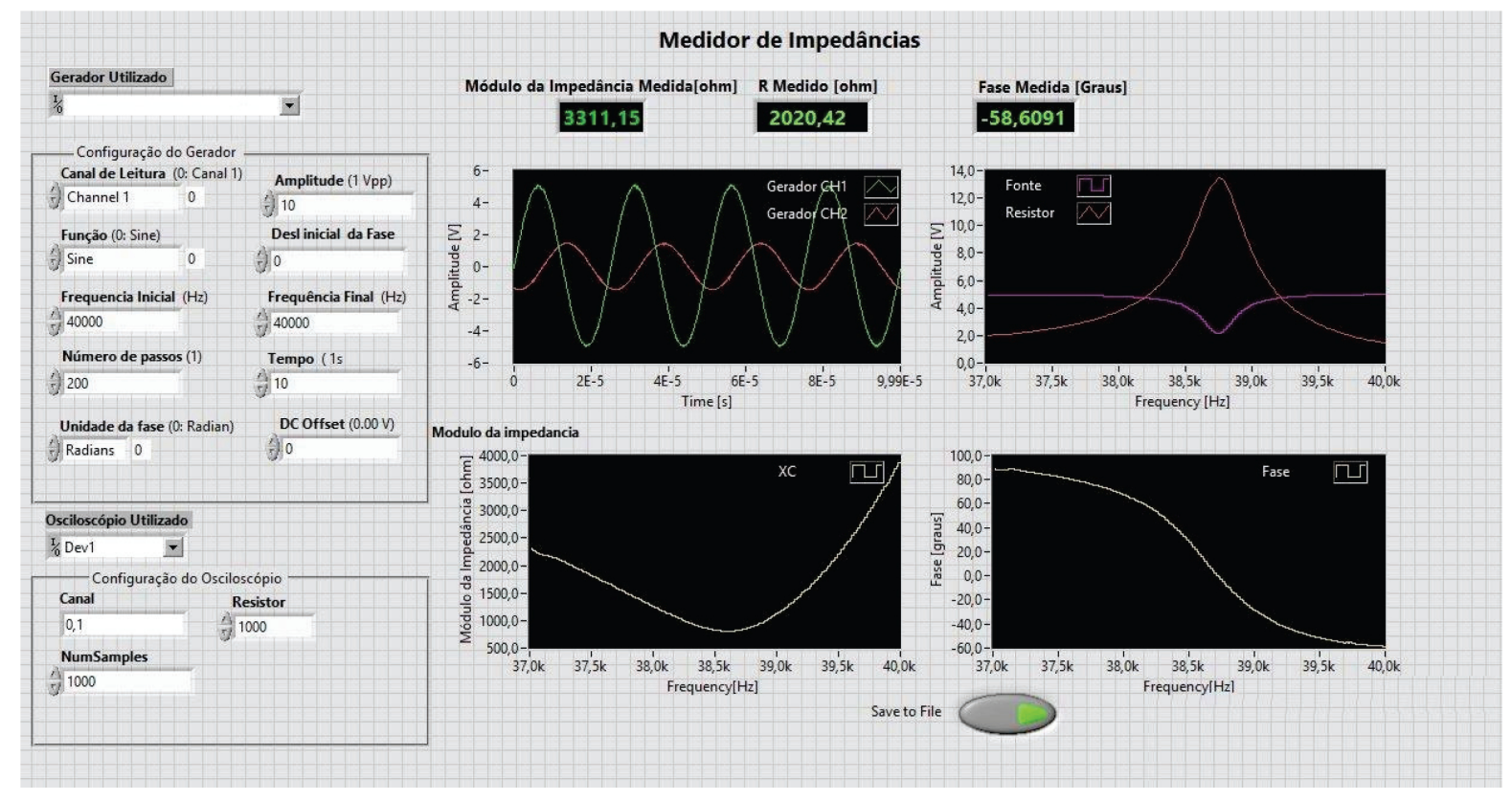

Figura 7: Interface do sistema de medições gerenciado pelo LabView. 

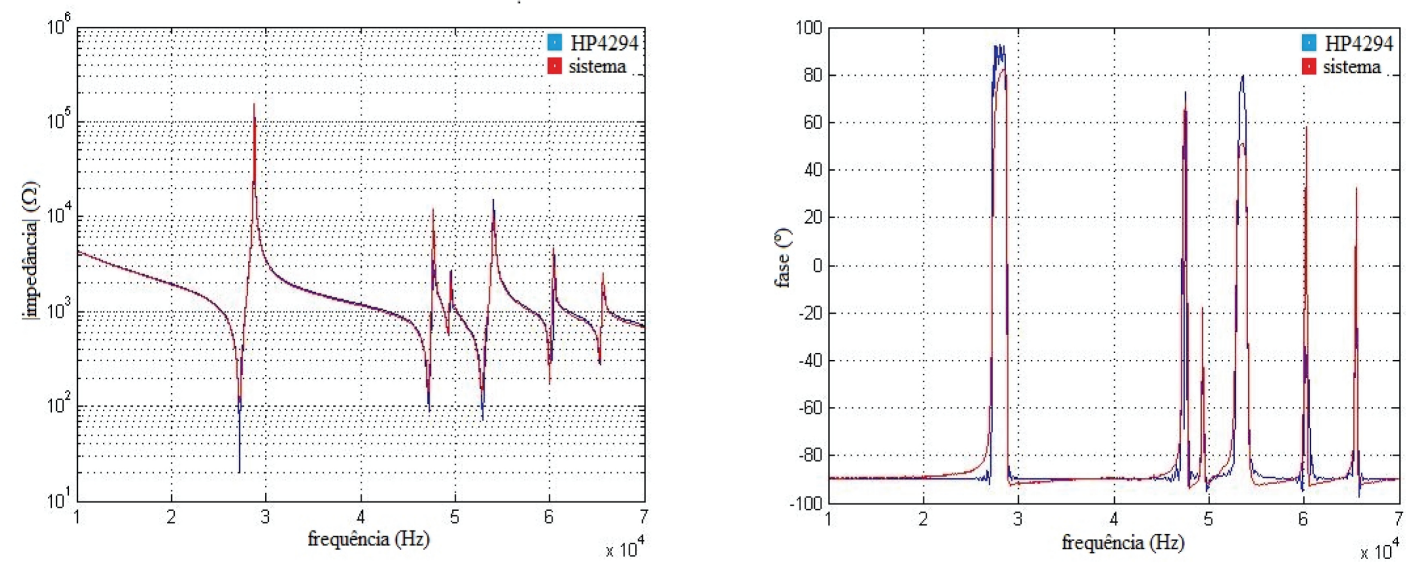

Figura 8: (a) Módulo da Impedância em função da frequência do transdutor P1. (b) Fase em função da frequência do transdutor P1. Em ambos os gráficos os traços azul e vermelho correspondem aos dados colhidos com o HP4292A e com sistema desenvolvido.
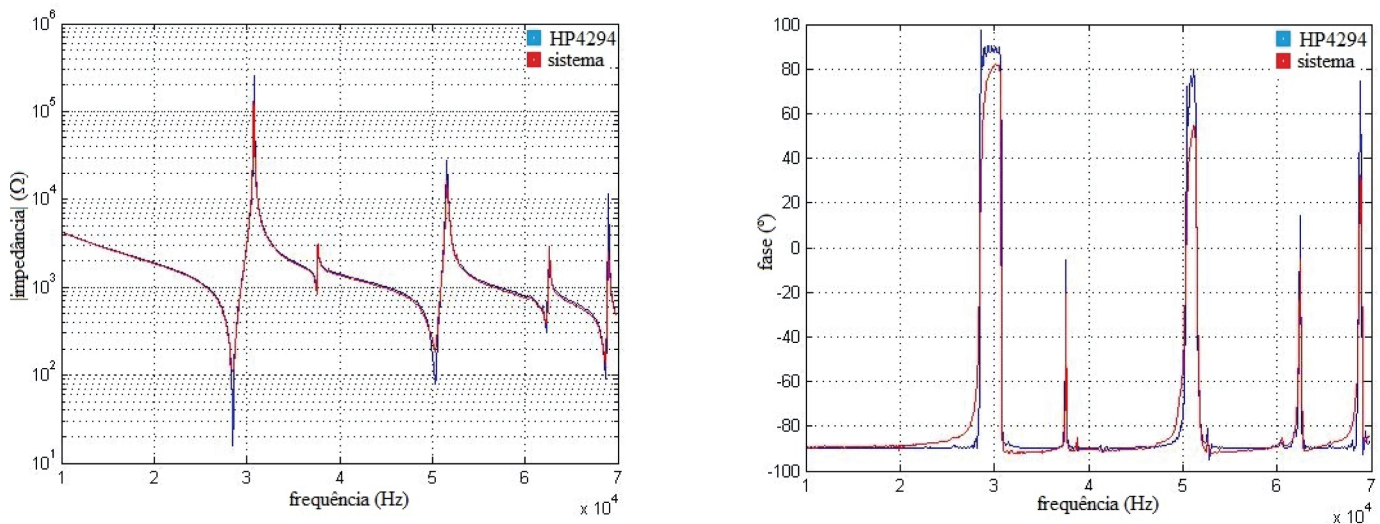

Figura 9: (a) Módulo da Impedância em função da frequência do transdutor P2. (b) Fase em função da frequência do transdutor P2. Em ambos os gráficos os traços azul e vermelho correspondem aos dados colhidos com o HP4292A e com sistema desenvolvido.
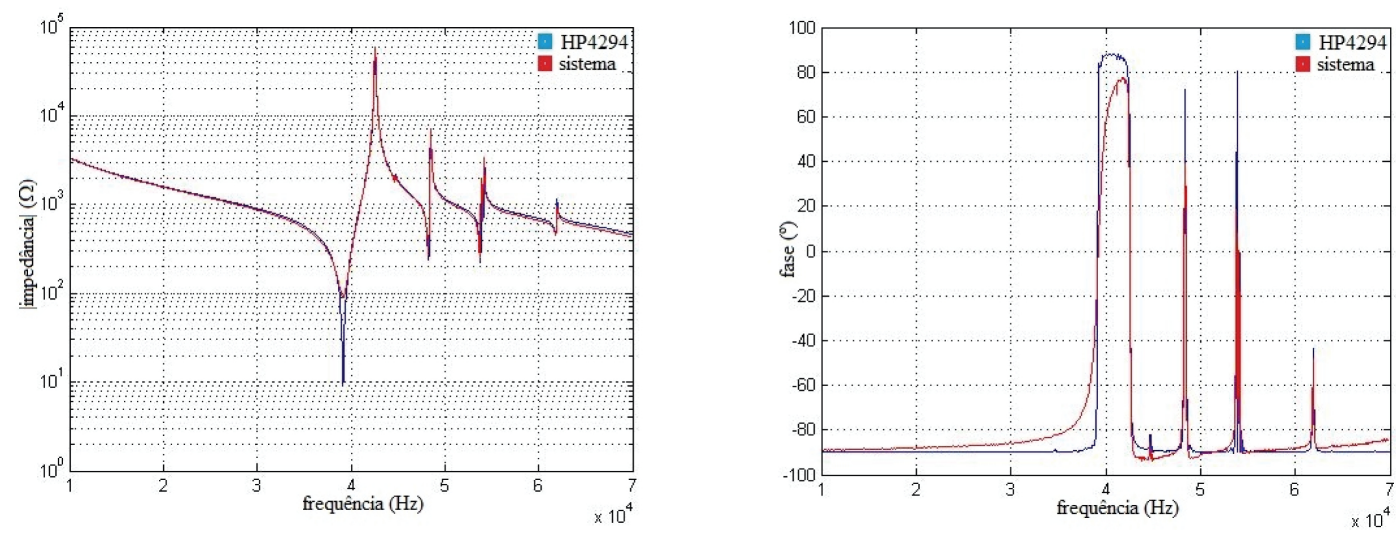

Figura 10: (a) Módulo da Impedância em função da frequência do transdutor P3. (b) Fase em função da frequência do transdutor P3. Em ambos os gráficos os traços azul e vermelho correspondem aos dados colhidos com o HP4292A e com sistema desenvolvido. 
elevação foi desempenhada satisfatoriamente pelo circuito desenvolvido. Portanto, a operação de transformação linear proposta contribui para o alargamento da faixa de valores do módulo da impedância a ser determinada.

Os resultados indicam que nos três transdutores piezelétricos testados o sistema desenvolvido pode medir magnitudes de impedâncias na faixa de dezenas de $\Omega$ a dezenas de $\mathrm{k} \Omega$, conforme proposto. Da mesma forma, as fases entre $-90^{\circ}$ e $90^{\circ}$ também foram obtidas.

Os erros médios relativos da magnitude (ERZ) e da fase $(E R \theta)$ calculados com as medições feitas com o sistema desenvolvido são apresentados na Tabela II. Notou-se que os erros apresentaram uma tendência de crescimento com a frequência. Em frequências mais elevadas os componentes do circuito, especialmente os resistores com valores da ordem de dezenas de $\mathrm{k} \Omega$ passam a apresentar efeito indutivo e, como consequência, progressivamente, geram imprecisões nas medições nas frequências superiores. A utilização de amplificadores operacionais de uso comum, como o TL084, também acarreta na redução da resposta na faixa de frequências. Havia inicialmente a pretensão de estender a utilização deste medidor de impedâncias até $100 \mathrm{kHz}$. Todavia, devido ao aumento dos erros a faixa de utilização recomendada ficou com limite superior de $70 \mathrm{kHz}$.

Intrinsecamente, a determinação da impedância elétrica de transdutores piezelétricos de alta potência é bastante crítica e pode contribuir para o aumento dos erros. Esses transdutores apresentam elevados valores de fator de qualidade, $Q$, portanto, em certas faixas de valores, pequenas diferenças nas frequências dos sistemas que produzirão as comparações dos valores podem gerar erros de elevada magnitude.

Foi verificado que o algoritmo para determinação do ângulo de fase entre dois sinais executado pelo LabView, que emprega ferramentas próprias desse software, apresenta deficiências quando sinais com pequena amplitude e apresentando distorções são submetidos. Isto explica o aumento dos erros na medição da fase nas proximidades das ressonâncias dos transdutores (valores mínimos de impedância).

Tabela 2: Erros médios relativos da magnitude ( $E R Z)$ e da fase $(E R \theta)$ dos transdutores P1, P2 e P3

\begin{tabular}{lccc}
\hline & P1 & P2 & P3 \\
\hline$E R Z(\%)$ & 7,41 & 5,63 & 9,64 \\
$E R \theta(\%)$ & 6,98 & 5,76 & 8,70 \\
\hline
\end{tabular}

A medição de impedâncias em transdutores piezelétricos de potência é um procedimento bastante crítico. Devido ao elevado fator $Q$ que esses transdutores apresentam, pequenas diferenças de frequência podem causar grandes discrepâncias entre os resultados. A automatização do sistema com o LabView permite mudar os passos da varredura em frequência e, assim, contribui para que os erros causados por esse motivo fossem minimizados.

Uma das vantagens da configuração proposta é a possibilidade de adequar a faixa de valores de impedância medidos alterando-se os valores de alguns resistores. Para tal, basta que se definam outros limites de tensão de saída e se refaçam os cálculos do sistema de equações (Equação 12). Também é possível usar este sistema em aplicações de campo, pois o gerador de funções, na forma de equipamento, utilizado neste trabalho pode ser substituído por um circuito integrado que realize a operação. A fonte de alimentação do sistema também pode ser substituída por baterias, uma vez que a corrente consumida é da ordem de dezenas de mA.

\section{Conclusões}

Foi apresentado um sistema para determinação de magnitude e fase da impedância elétrica de transdutores piezelétricos. O artifício utilizado para superar dificuldades de medição de valores extremos mostrou-se satisfatório em termos comparativos com os resultados obtidos com um analisador de impedâncias comercial. A forma com que o projeto foi desenvolvido pode ser replicada para outras faixas de valores de magnitude da impedância e outras frequências. Para tal, basta estabelecer os parâmetros e utilizar componentes semicondutores adequados para operações nas faixas de frequência e impedâncias elétricas consideradas.

Em relação aos custos, o módulo digital, a licença do software (LabView) e os componentes utilizados no circuito totalizam, aproximadamente, 3000 dólares. No entanto, este valor pode ser amortizado pois o módulo digital e o software podem ser partilhados na automatização de outros experimentos de um laboratório de física. Sendo assim, comparado ao valor de um analisador de impedâncias comercial (um HP4294A) que é de aproximadamente 45000 dólares, pode-se dizer que o custo do dispositivo é muito baixo. 
Há possibilidades de aperfeiçoamento do sistema com o uso de resistores de precisão e amplificadores operacionais que respondam em frequências mais elevadas. Com a inserção desses componentes espera-se produzir sensível melhoria na precisão, principalmente nas frequências superiores a $70 \mathrm{kHz}$, em que a taxa de erro na curva de fase foi maior.

Embora os testes tenham sido realizados em transdutores piezelétricos, o sistema apresentado pode ser empregado para a prática de aulas experimentais de Fisica IV em que se aborda a análise de circuitos em correntes alternadas com componentes resistivos e reativos e circuitos ressonantes. Por ser automatizada, a configuração proposta permite que experimentos, como o proposto em [23], possam ser reproduzidos com maior celeridade e dinamismo na obtenção de dados.

\section{Agradecimentos}

Os autores agradecem a Fundação de Amparo à Pesquisa do Estado de São Paulo (FAPESP) e ao Conselho Nacional de Pesquisa (CNPq) pelo apoio financeiro dado a realização deste trabalho.

\section{Referências}

[1] J.C. Andrades, A. Schiappacassa e P.F. Santos, Rev. Bras. Ens. Fis. 35, 2503 (2013).

[2] L.A. Dworakowski, A.M. Hartmann, E.M. Kakuno e P.F.T. Dorneles, Rev. Bras. Ens. Fis. 38, e3503 (2016).

[3] F.J. Arnold, R. Arthur, L.L. Bravo-Roger, M.S. Gonçalves e M.J.G. Oliveira, Rev. Bras. Ens. Fıs. 33, 4311 (2011).

[4] C. Sirisathitkul, P. Glawtanong, T. Eadkong and Y. Sirisathitkul, Rev. Bras. Ens. Fis. 35, 1504 (2013).

[5] A.G. Guedes, Rev. Bras. Ens. Fis. 37, 2502 (2015).

[6] P.F.O. Rosa, F.S. Silva, L. Benyosef e A.R.R. Papa, Rev. Bras. Ens. Fis. 38, 1501 (2016).

[7] A.C.P. Fernandes, L.T.S. Auler e J.A.O. Huguenin, Rev. Bras. Ens. Fis. 38, e3504 (2016).

[8] D. Halliday, R. Resnick e J. Walker, Fundamentos de Física (LTC, Rio de Janeiro, 2012), 9 ${ }^{\mathrm{a}} \mathrm{ed}$, vol. 4.

[9] P.A. Tipler e G. Mosca, Física para Cientistas e Engenheiros (LTC, Rio de Janeiro, 2012), $6^{\text {a }}$ ed, vol. 2.

[10] P.R. Kesten e D.L. Tauck, Física na Universidade para as Ciências Físicas e da Vida (LTC, Rio de Janeiro, 2015), $1^{\mathrm{a} e d,}$ vol. 3.

[11] J.W. Dally, W.F. Riley and K.G. McConnell, Instrumentation for Engineering Measurements (John Wiley \& Sons, New York, 1984)
[12] A. Saffari and E.K. Akdogan, Piezoelectric and Acoustic Materials for Transducer Applications (Springer, New Jersey, 2008).

[13] F.J. Fishman, Am. J. Phys. 55, 1047 (1987).

[14] D.M. Peairs, G. Park and D.J. Inman, J. of Intell. Mat. Systems and Struct. 15, 129 (2004).

[15] F.G. Baptista, and J. Vieira Filho, IEEE Trans. on Instrum. and Meas. 58, 3602 (2009).

[16] S. Wang, and C. You, J. of Intell. Mat. Systems and Struct. 19, 1029 (2008).

[17] F.J. Arnold, R.L. Ximenes, R. Arthur e T.S. Santos, in: Anais do XX Congresso Brasileiro de Automática p. 1712-1717 (2014).

[18] S. Doerner, T. Schneider and P.R. Hauptmann, Rev. of Sci. Instrum. 78, 105101 (2007).

[19] M. Schmid, E. Benes and R. Sedlaczek, Meas. Sci. Tech 1, 970 (1990).

[20] J.A. Edminister, Circuitos Elétricos (Editora McGraw-Hill, São Paulo, 1991), $2^{\mathrm{a}}$ ed.

[21] R.L. Boylestad, Análise de Circuitos (Editora Pearson, São Paulo, 2012), $12^{\mathrm{a}}$ ed.

[22] A. Pertence, Amplificadores Operacionais e Filtros Ativos (Makron Books, São Paulo, 1996), $5^{\mathrm{a}}$ ed.

[23] D.L. Chinaglia, G. Gozzi, R.A.M. Alfaro, e R. Hessel, Rev. Bras. Ens. Fis. 30, 4504 (2008). 